Worryingly, the QOF statements relating to lithium treatment have now been retired as of April 2019. It is suggested that psychiatrists are aware of the challenges primary care faces when monitoring lithium treatment.

\section{Consultation liaison to support efficient delivery of mental health care}

Emma Davies* and Mihaela Bucur

Sussex Partnership NHS Foundation Trust

${ }^{\star}$ Corresponding author.

doi: 10.1192/bjo.2021.835

Aims. To study the impact of collaborative working, via consultation liaison, between Mental Health Liaison Practitioners (MHLPs) and Doctors within a secondary care mental health service. We hypothesise that this model of working may avoid unnecessary clinic appointments and waiting times, whilst providing patients with more efficient treatment.

Background. Mental health services are stretched, understaffed and under-resourced. It is estimated that $75 \%$ of people with mental health problems in England may not get access to the treatment they need. We therefore need efficient and innovative ways for people who seek help to receive support. Good practice consultation liaison involves face to face contact between clinicians; treatment can be delivered by supporting primary care whilst reducing the burden of secondary care mental health services.

Method. Regular 30-minute sessions within an Assessment and Treatment Service, between MHLPs and Doctors, at both Consultant and Trainee level, were coordinated. Patients assessed by MHLPs were discussed by opening a dialogue whereby further management was discussed across a multi-professional team. A record was created of all patients discussed and the outcome.

Result. Number of MHLP/Doctor sessions: 10 across a six-month period.

Number of patients discussed: 17.

Medication advice provided for 16 patients. One patient required a referral for a clinic appointment.

For several patients, integrated working procured alternative care pathways and resources to be considered, to incorporate into individual treatment plans.

Conclusion. Regular consultation liaison with MHLPs and Doctors is a model of working across the interface between primary care and specialist mental health services. It may provide patients with more efficient care, whilst avoiding unnecessary waiting times for clinic appointments. The consultation liaison working supported the development of an educative relationship between clinicians, with interprofessional learning. This is an example of an integrated and collaborative care model, whereby multi-professional working can provide efficient and effective treatment, whilst the support for the patient can remain in the primary care setting.

\section{Physical health monitoring in antipsychotic depot clinic}

Rebecca Davies ${ }^{1 \star}$, Anu Priya ${ }^{1}$, Hardev Bhogal ${ }^{1}$, Adesola Omodara ${ }^{1}$, George Davies ${ }^{2}$ and Shweta Mittal ${ }^{1}$

${ }^{1}$ Nottinghamshire Healthcare NHS Foundation Trust and

${ }^{2}$ Nottingham Medical University

${ }^{\star}$ Corresponding author.

doi: 10.1192/bjo.2021.836
Aims. A service evaluation project to look at if annual bloods, ECG, physical examination, and medical review was completed within the last year for patients attending anti-psychotic depot clinic at Bassetlaw mental health services in Nottinghamshire HealthCare NHS Foundation Trust.

Method. Electronic notes were examined in October 2020 for 25 patients who attend anti-psychotic depot clinic to ascertain if medical review and physical examination had been completed along with annual bloods and ECG.

Result. Out of 25 patients attending depot clinic in 2020 at Bassetlaw Hospital, 21 had all their blood tests done, 1 patient had refused bloods and 2 patients did not have blood tests done. ECG was completed for 3 patients at Bassetlaw hospital and 8 patients had it requested from primary care with 2 patients refusing to have ECG done. For 12 patients there was no evidence of ECG being requested or completed. 8 patients had physical examination completed and rest 17 patients did not have the physical examination completed including due to refusal. Out of 25 , only 14 patients had a medical review conducted.

Conclusion. Patients who attend depot clinic may have an allocated community psychiatric nurse $(\mathrm{CPN})$ or get reviewed by medics in outpatient clinics and would usually have their blood tests, physical health examination and ECGs requested and monitored by them. Patients who do not have any allocated CPN or medic tend to miss out on blood tests and ECG. General Practitioners are expected to complete physical health checks for patients who do not have CPN or regular outpatient review. The results of these investigations may not always be received in depot clinic, hence there is no documentation on electronic RIO system. When these patients disengage from the depot clinic, it is often very difficult to track them. As a follow-up from this service evaluation, all depot clinic patients will be allocated a key worker/CPN. This will ensure that they have a responsible person to facilitate annual checks. This will be reviewed in a years' time to evaluate the effectiveness of this intervention.

\section{Re-evaluating trainee experience of involvement in serious incidents - has anything changed?}

Glori-Louise de Bernier ${ }^{\star}$, Alice Debelle, Marilia Calcia and Lauren Waterman

South London and Maudsley NHS Foundation Trust ${ }^{\star}$ Corresponding author.

\section{doi: 10.1192/bjo.2021.837}

Aims. To complete an audit cycle to investigate: trainees' experiences of SI involvement since 2017, perceptions of current support systems and trust facilitation of learning from SIs and the impact of the interventions implemented following the 2017 survey.

Background. In 2017, data were collected from trainees working in psychiatry within two London trusts to examine the nature of their involvement in serious incidents (SIs), their experience of the process following an SI and their knowledge of the support systems available to them. Due to concerning results from this, several interventions were put in place in accordance with trainees' suggestions.

Method. Cross-sectional surveys were e-mailed to trainees of all grades in July 2019, including GP and foundation doctors, working within two mental health trusts. These built upon the 2017 surveys, additionally enquiring about demographic information and the personal and training consequences of SIs on trainees.

Result. 61 (15\% of all trainees) returned the survey with 41 (67\%) respondents unable to recall any SI related teaching during induction and $47(77 \%)$ not having received a written guidance document on SI procedures. 
24 (39\%) had been involved in an SI. Only half felt adequately supported by the trust at internal investigation. Knowledge of the available internal and external sources of support ranged from $38-71 \%$ however these sources were rarely utilised. 12 (60\%) trainees did not feel that learning had been facilitated following an SI and almost none had been informed of internal investigation outcomes.

Respondents who gave a low (1-4/10) rating of support from their NHS Trust were more likely to have been informed about the incident in person, been invited to team-based support or been aware of the variety of sources of support available, when compared with respondents who scored their Trust support more highly. Suggestions for improvements made by trainees included opportunities to observe coroners' inquests and a peer support scheme from colleagues with experience of SI involvement.

Conclusion. Unfortunately, trainees did not report much improvement in their experiences compared those in the 2017 survey, and a large proportion continued to feel unsupported. Interventions had not been as widely circulated as intended and only half of trainees had been invited to team-based support. Possible further interventions include increasing email communication to trainees following SIs and setting up a peer support scheme. We are in the process of organising a coroner's inquest observation programme for trainees.

\section{Adherence to Public Health England (PHE) guidance} for the use of personal protective equipment (PPE) in north Wales mental health unit- a regional audit

Asha Dhandapani*, Sathyan Soundararajan, Alberto Salmoiraghi, Shona Ginty, Tajnin Mitu, Justina Akinlua, Catrin Thomas, Rahul Malhotra, Zeenish Azhar, Haseeb Bhutta, Hanani Taib, Nikhil Gauri Shankar, Vikram Bhangu, Gathoni Kamau, Elizabeth Chamberlain, Anna Mackenzie, Henrik PAHLEN, Hannah Lock, Aniis Rymansaib, Pauline Mclean, Rodrigo Trujillo, Manjula Simiyon, Adam Chappell, Agnieszka Gross and Gaynor Gaskell

BCUHB

${ }^{\star}$ Corresponding author.

doi: 10.1192/bjo.2021.838

Aims. To ensure that the PPE guidance is strictly adhered to.

To ensure that patient care is not compromised.

To help us in areas of need in order to educate the staff regarding the techniques of PPE and thus ensure patient and staff safety and care during the pandemic.

Method. Novel coronavirus 2019 was first described in December 2019 in Wuhan in China. Since those initial few cases, it has rapidly proliferated to a global pandemic, putting an inordinate amount of strain on healthcare systems around the world. We believe that the technique of donning and doffing if followed as per PHE guidelines would be of help in both preventing the infection and improve the care and safety of both patients and staff.

This Audit includes both In-patient and Out-patient units in Psychiatric services across North Wales. Data were collected from 19 units out of 39 . We observed covertly 325 staff members belonging to various cadres. Apart from the Donning and Doffing techniques, we also observed the availability of designated areas for this purpose and the availability of PPE as well.

Data collection was by junior and senior doctors from various sites of the mental health unit in North Wales. A proforma was provided, the standards were based on PHE guidelines.

Result. It was noted that just about $50 \%$ of the staff followed donning as per guidance. Amongst all three sites, the Central team showed a better adherence with $85 \%$ of them donning PPE correctly. whereas only $22 \%$ adhered to donning in the West team.

Only $21 \%$ of them managed to doff PPE as per guidance amongst all 3 centres in North Wales.

It was also noted that there are no designated areas to Don and Doff in outpatient units. Staff, in general, seem to not adhere to the guidance of utilising a mask, especially when within 2 meters distance of other staff.

Conclusion. We will be presenting the Audit at the regional meeting. After discussion with the infection prevention control team and Health and safety lead, we intend to improvise the wards with designated areas for donning and doffing. Teaching sessions for the staff in all three sites, reminders in various areas of the community mental health units and inpatient units.

We are hoping that these recommendations will help us in achieving our aim of health and safety during this pandemic.

\section{A retrospective analysis comparing clinical outcome} measures pre- and post- the introduction of telehealth in a community-based psychiatry clinic in a tertiary medical centre

Arup Dhar ${ }^{1 \star}$, Liam Edwards ${ }^{2}$ and Moana Waerea ${ }^{3}$

${ }^{1}$ Monash Health, Department of Psychiatry, Monash University, Faculty of Medicine, Nursing and Health Sciences; ${ }^{2}$ Monash

University, Faculty of Medicine, Nursing and Health Sciences and

${ }^{3}$ Monash Health, Department of Psychology

${ }^{\star}$ Corresponding author.

doi: 10.1192/bjo.2021.839

Aims. The aim of this retrospective analysis was to look at the effect that telehealth had on patient outcomes and the therapeutic alliance. Method. Clinical outcomes measures were collected prospectively as part of routine clinical care. Outcome measures were administered at patients' initial and final appointment. Information was merged into a single database and imported into IBM SPSS for retrospective analysis. The following measures were administered at the beginning and end of treatment and were used to evaluate patient progress; Health of the Nation Outcome Scale (HoNOS), Life Skills Profile (LSP), Session Rating Scale (SRS), Outcome Rating Scale (ORS).

Result. Two cohorts were derived from the clinic; the first cohort ( $\mathrm{n}=90 ; 53$ females; 37 males; $\mathrm{M}=35.72$ years; $\mathrm{SD}=12.12$ years) comprised of those patients whose care occurred between 23/09/ 2019 and 22/03/2020 and did not receive telehealth appointments. The second cohort ( $\mathrm{n}=122$; 68 females; 54 males; $\mathrm{M}=36.2$ years; $\mathrm{SD}=12.78$ years) were those patients who presented to the clinic and were discharged between 23/03/2020 and 21/09/2020 and received at least one telehealth appointment. In the pre-telehealth cohort, mean HoNOS scores at baseline were 17.87 compared to 13.53 at discharge, mean LSP scores at baseline were $10.76 \mathrm{com}$ pared to 9.01 at discharge, mean SRS scores at baseline were 34.17 compared to 36.04 at discharge, and mean ORS scores at baseline were 12.97 compared to 21.28 at discharge. In the posttelehealth cohort, mean HoNOS scores at baseline were 14.45 compared to 10.50 at discharge, mean LSP scores at baseline were 7.85 compared to 7.19 at discharge, mean SRS scores at baseline were 36.04 compared to 35.36 at discharge, and mean ORS scores at baseline were 18.83 compared to 15.85 at discharge.

Conclusion. Results show that telehealth did not impact negatively on the therapeutic effect of clinical sessions, highlighted by similar reductions in HoNOS and LSP scores. It was seen in the post-telehealth cohort that there was worsening in the subject-rated scales (SRS and ORS) which was not seen in the pretelehealth face-to-face cohort. Thus, there seems to be a 